\title{
Uma Solução Parcial Para o Lixo Eletrônico: Geração de Energia Elétrica e Conservação do Meio Ambiente.
}

\author{
Ana Carolina Maistrovick ${ }^{1}$; Fernando da Silva Alves²; Roger Nabeyama Michels ${ }^{3}$; Ivan Marcelo Laczkowski ${ }^{4}$, \\ Manoel Messias Alvino de Jesus ${ }^{5}$.
}

High School student - Ku Pao Home Economics and Comercial High School - Taipei/Taiwan

Físico, Doutor em Física, Professor da UTFPR-Londrina/PR

Tecnólogo em Eletromecânica, Mestre em Eng. Agrícola, Professor da UTFPR-Apucarana/PR; rua Marcílio Dias, 635, CEP 86812-460, Apucarana-PR.rogernmichels@utfpr.edu.br;

Físico, Doutor em Física, Professor da FECILCAM-Campo Mourão/PR;

Físico, Doutor em Física, Professor da UTFPR-Apucarana/PR;

\section{RESUMO}

Nesse trabalho é apresentada uma proposta de solução parcial para o lixo eletrônico criado a partir de computadores sucateados, utilizando motores de imã permanente como geradores eólicos de eletricidade. Tais geradores foram criados a partir do uso de motores de imã permanente contidos nos drives de leitura e gravação de CD e DVD, usando o benefício do fenômeno da indução eletromagnética. Doze motores de imãs permanentes foram associados em série, de forma que a diferença de potencial elétrico (ddp) gerada foi resultado da soma das diferenças de potenciais geradas por cada um dos motores. As ddp's geradas variaram entre zero e $30 \mathrm{~V}$ com potencia variando entre $0 \mathrm{~W}$ e $297 \mathrm{~mW}$. Pode-se concluir que o uso de motores de imã permanente obtidos de lixo eletrônico resulta em geração de energia elétrica diminuindo o impacto ambiental que esse tipo de resíduo provoca na natureza.

Palavras chave: Energia elétrica, lixo eletrônico.

\section{A partial solution to the electronic junk: Electricity Generation and Environmental Conservation.}

\begin{abstract}
This work presents a proposal for a partial solution to electronics junk created from scrapped computers, using permanent magnet motors as wind generators of electricity. Such generators have been created from the use of permanent magnet motors contained in CDs and DVDs read and write drives, using the benefit of the electromagnetic induction phenomenon. Twelve permanent magnets motors were associated in series so the electrical potential difference (epd) generated was a result of the potential differences generated sum of each motor. The epd's generated ranged between zero and $30 \mathrm{~V}$ with power ranging from $0 \mathrm{~W}$ to $297 \mathrm{~mW}$. It can be concluded that the use of permanent magnet motors obtained from electronic junk results in electricity generation, reducing the environmental impact that this type of waste causes in nature.
\end{abstract}

Keywords: electricity, electronic junk. 


\section{INTRODUÇ̃̃O}

A velocidade no desenvolvimento de produtos tecnológicos vem se acentuando ano a ano. Todos os dias surgem novas máquinas e produtos eletrônicos criados para facilitar a vida das pessoas, seja em suas casas, seja em seus ambientes de trabalho .

Por um lado, pesquisadores e engenheiros das mais diversas áreas se preocupam com a melhora no bem-estar e a comodidade das pessoas. Por outro lado, empresas de bens de consumo e serviços preocupam-se cada dia mais em aumentar seus lucros. O resultado dessa complexa combinação é um momento onde a tecnologia começa a deixar de ser solução para a melhoria na qualidade de vida das pessoas, tornando-se um problema ambiental de difícil solução (REVISTA DO IDEC, 2009).

Todos os anos são lançados novos modelos de refrigeradores, televisores, máquinas de lavar roupa e, sobretudo, computadores.

O caso específico dos computadores é especialmente grave, sobretudo porque a cada minuto um novo software é desenvolvido e exige máquinas cada vez mais velozes e especificas para suas aplicações. Isso tem reduzido drasticamente o tempo de uso de desktops e leptops (REVISTA DO IDEC, 2009).

Uma solução paliativa é o reuso e aproveitamento de maquinas, obsoletas, para algumas utilidades ainda viáveis em ambientes de trabalho ou domestico, que não exijam alto grau de desenvolvimento desses materiais.

O problema surge quando não existe mais utilidade alguma e, sobretudo, quando não existe conserto para defeitos específicos. Nesses casos, que destino deve ser dado para esse tipo de lixo tecnológico? Não existem depósitos especiais para esse tipo de lixo e o abandono em lixões tradicionais e até mesmo em terrenos baldios tem sido a prática mais comum.
De fato, um computador sucateado, pode ser totalmente reciclado, com um pouco de paciência e mão de obra qualificada. Nesse caso, o aproveitamento do material constituinte da máquina passa, necessariamente, por um profissional mais qualificado que os coletores e trabalhadores de materiais reciclados tradicionais, encarecendo drasticamente o processo (REVISTA DO IDEC, 2009).

Admitindo que os benefícios, para o meio ambiente, gerados pela reciclagem desses materiais sejam superiores aos investimentos necessários para qualificar mão de obra especializada, esse trabalho apresenta uma solução parcial para o lixo eletrônico gerado por computadores sucateados.

Nesse caso, é apresentada uma proposta de construção de geradores eólicos de eletricidade, criados a partir do uso de motores de imã permanente contidos nos drives de leitura e gravação de CD e DVD, usando o benefício do fenômeno da indução eletromagnética descoberto por Faraday (DIAS \& MARTINS, 2004).

\section{FUNDAMENTAÇÃO TEÓRICA}

O fenômeno da indução eletromagnética foi descoberto pelo físico inglês Michael Faraday em 1831 (DIAS \& MARTINS, 2004).

Se uma bobina condutora, pela qual circula uma correte elétrica, for colocada em uma região onde atua um campo magnético, um torque mecânico atuará sobre ela, podendo fazê-la girar (DIAS \& MARTINS, 2004), (SOUZA FILHO et al, 2007). Dessa forma, existe uma transformação de energia elétrica (ou eletromagnética) em energia mecânica (SILVEIRA \& VARRIALE, 2009).

De maneira análoga, um torque mecânico em uma bobina condutora, provoca o aparecimento de uma corrente elétrica nessa bobina, se a mesma estiver colocada em uma região de campo magnético. Esse é o princípio do gerador elétrico, e o fenômeno do surgi- 
mento da corrente elétrica é conhecido como principio da indução elétrica, cuja interpretação está ancorada na Lei da Indução de Faraday. (WALKER, 1998; DIAS \& MARTINS, 2004), de acordo com a qual: "A tensão elétrica induzida numa espira condutora é igual ao negativo da taxa de variação do fluxo magnético ao qual ela está submetida".

Matematicamente, essa Lei pode ser escrita na forma:

$$
V=-\frac{d \phi_{B}}{d t}
$$

Para "N"espiras:

$$
V=-N \frac{d \phi_{B}}{d t}
$$

Onde V é a força eletromotriz induzida.

\section{DESENVOLVIMENTO}

Os geradores elétricos eólicos foram montados com materiais remanescentes de obras de construção civil e motores de imã permanente. $\mathrm{O}$ processo de montagem dos geradores são ilustrados nas figuras 1 a 8 .

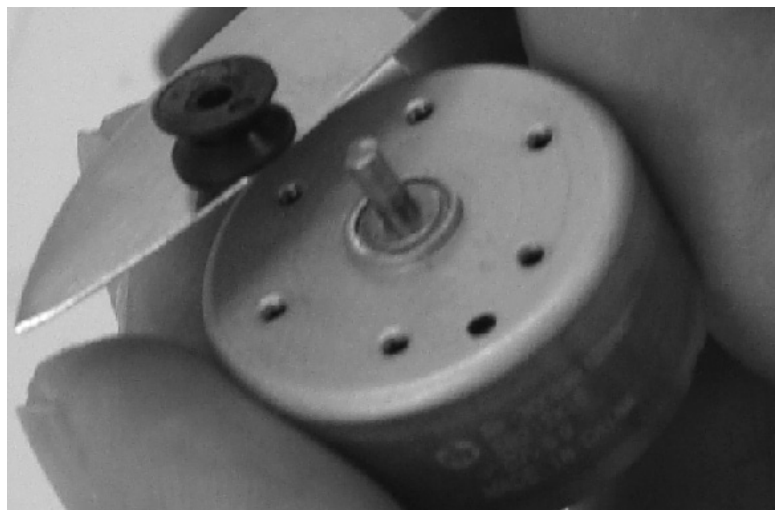

Figura 1 - motor utilizado

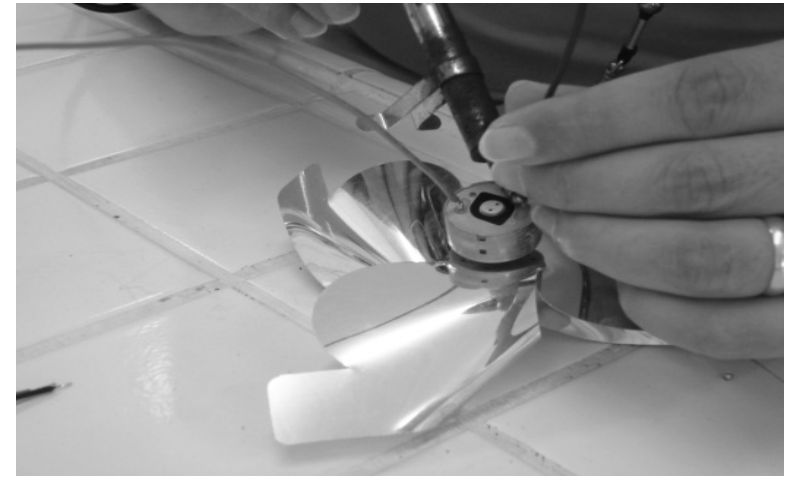

Figura 2 - Colocação da hélice.

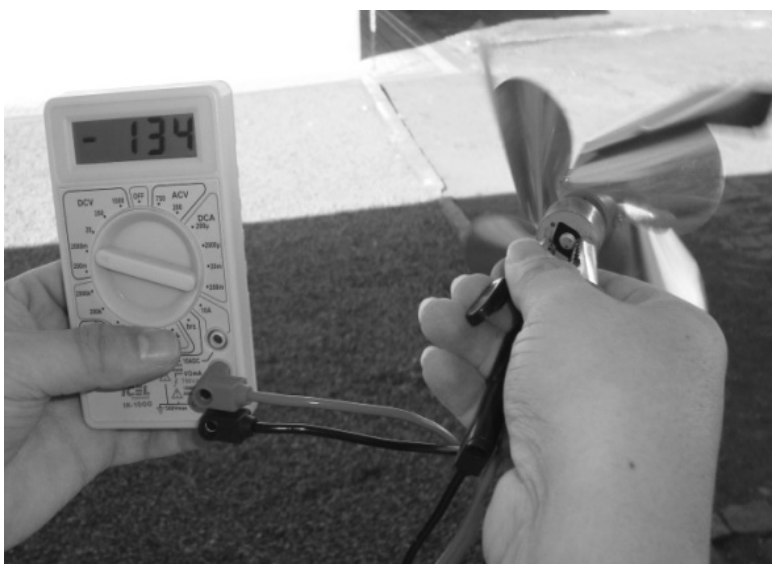

Figura 3 - Teste de geração de energia elétrica.

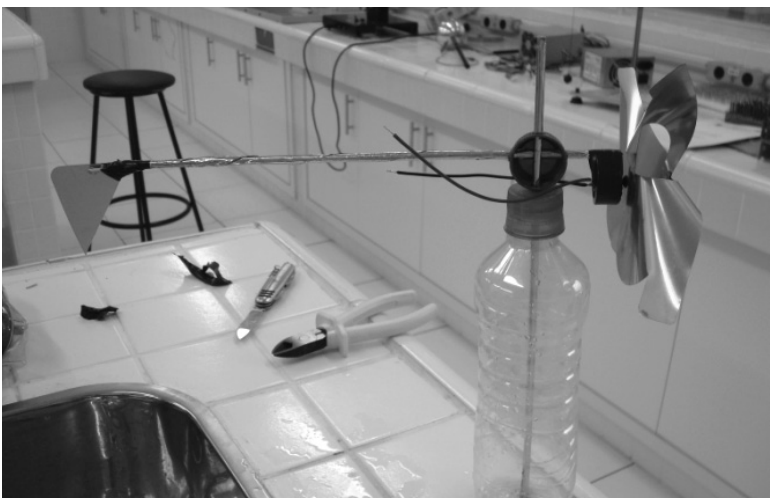

Figura 4 - Protótipo 1. 


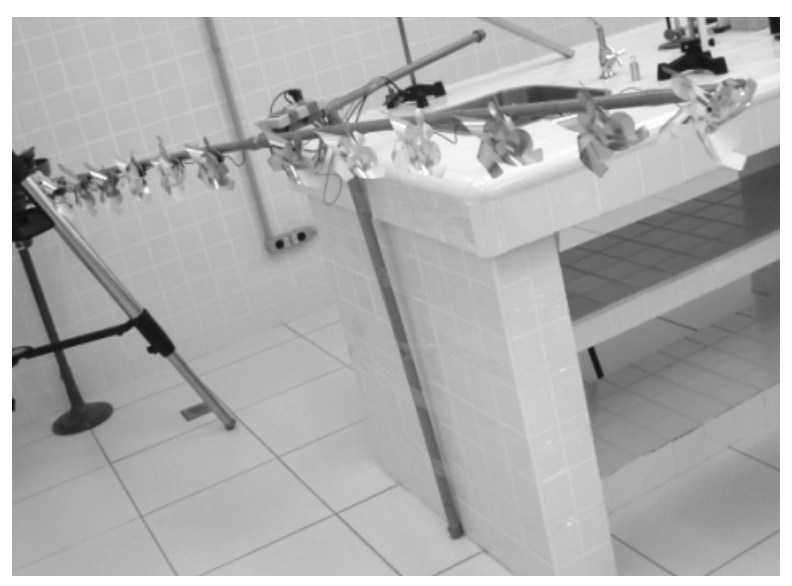

Figura 5 - Protótipo 2.

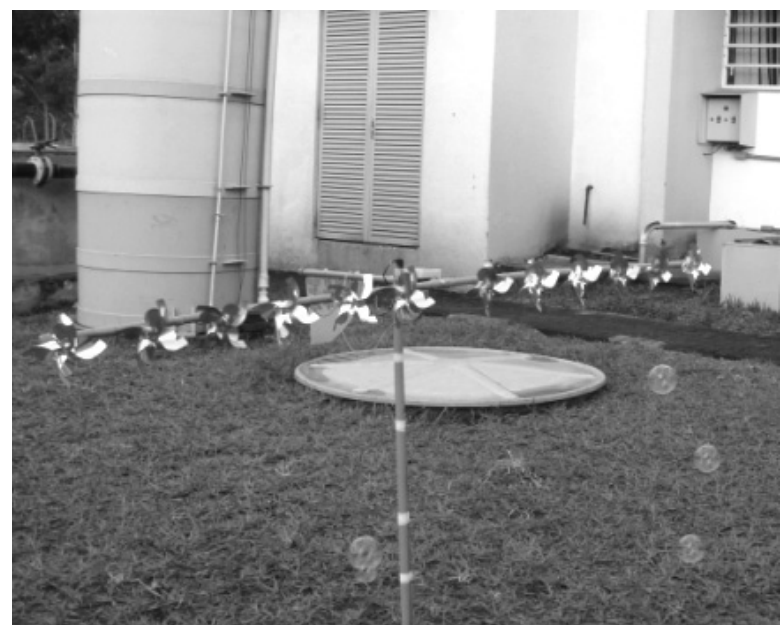

Figura 6 - Teste de funcionamento e orientação na direção do vento.

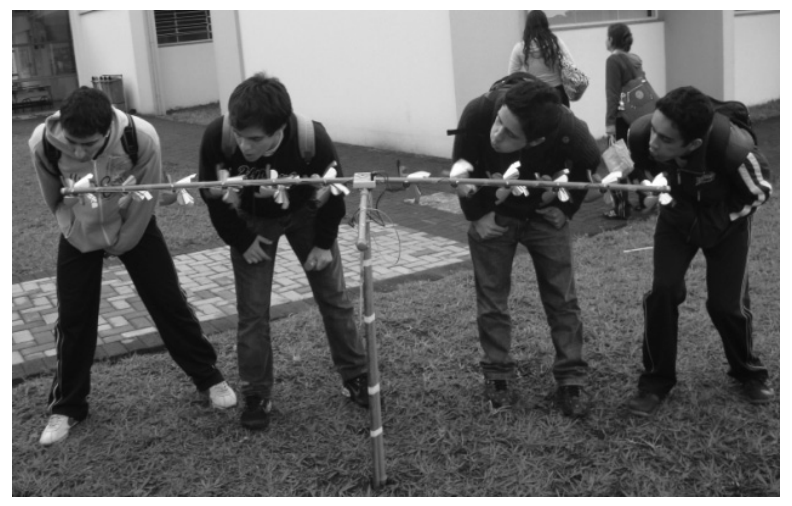

Figura 7 - Teste de funcionamento e orientação.

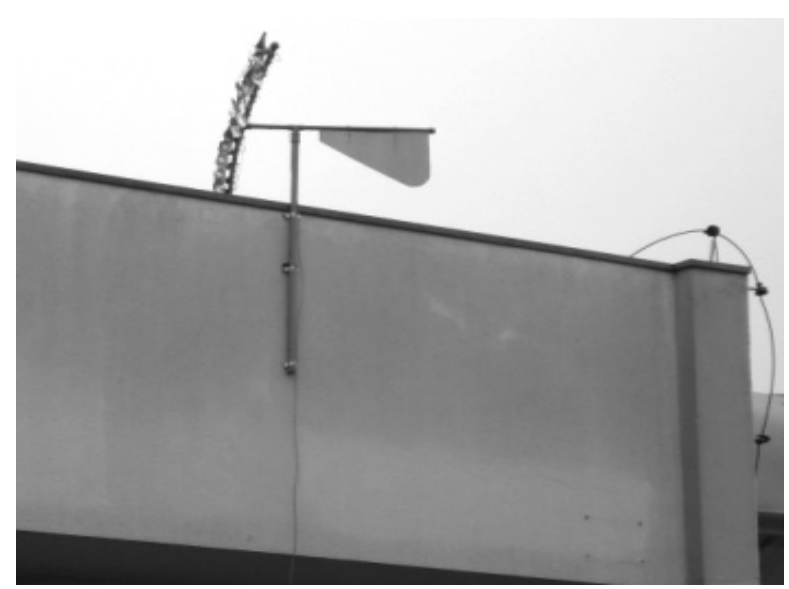

Figura 8 - Aspecto final do protótipo instalado.

Como se observa na figura 8 , o impacto na fachada do prédio no qual foi fixado o protótipo final não foi drasticamente alterado

\section{RESULTADOS}

Um total de doze motores de imãs permanentes foram associados em série, de forma que a diferença de potencial elétrico gerada foi resultado da soma das diferenças de potenciais geradas por cada um dos motores.

As ddp's geradas variaram entre zero e 30 volts com potencia variando entre $0 \mathrm{~W}$ e $297 \mathrm{~mW}$. Se considerarmos uma situação de potencia máxima gerada, ao longo de 1 dia, poderia ser gerado um trabalho de 0,00713 kWh. Em um mês, 0,21 kWh. Em um ano, resultaria em uma geração de $2,56 \mathrm{kWh}$ e um resultado financeiro de aproximadamente $\mathrm{R} \$ 1,20$.

De fato, o resultado financeiro não é um dos melhores, mas se for levado em consideração o impacto ambiental, é fato que o projeto é viável.

As figuras 9 e 10 ilustram o aparato experimental utilizado e a forma de obtenção dos dados. 


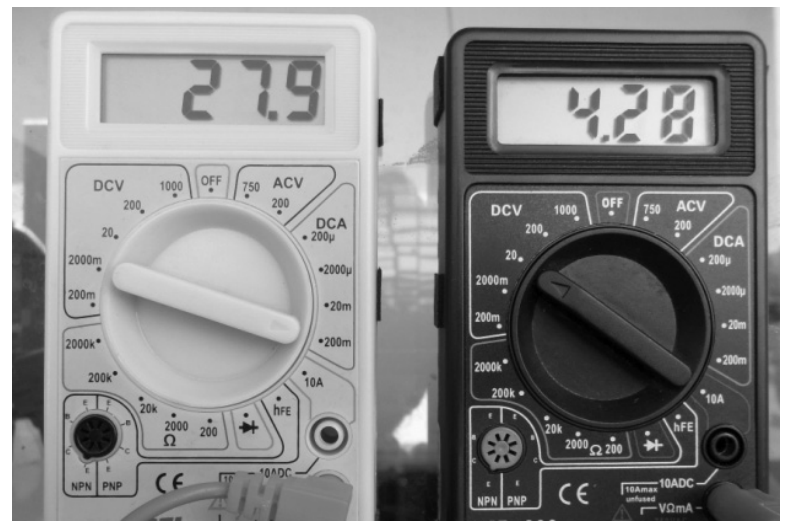

Figura 9 - Aquisição de dados.

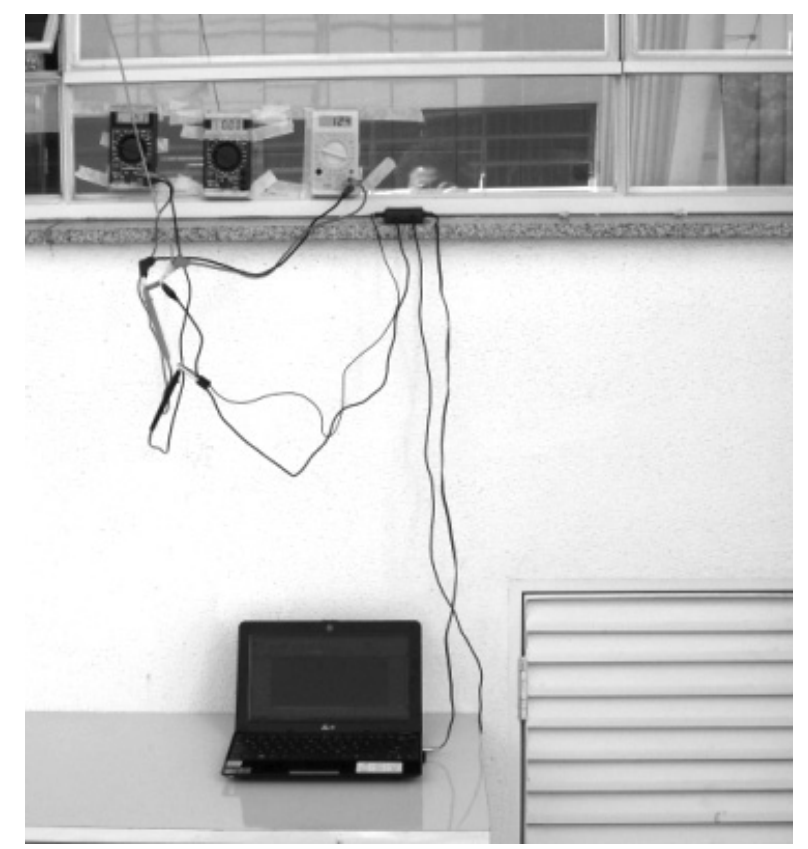

Figura 10 - Aquisição de dados.

A figura 11 ilustra um exemplo de uso da energia elétrica gerada.

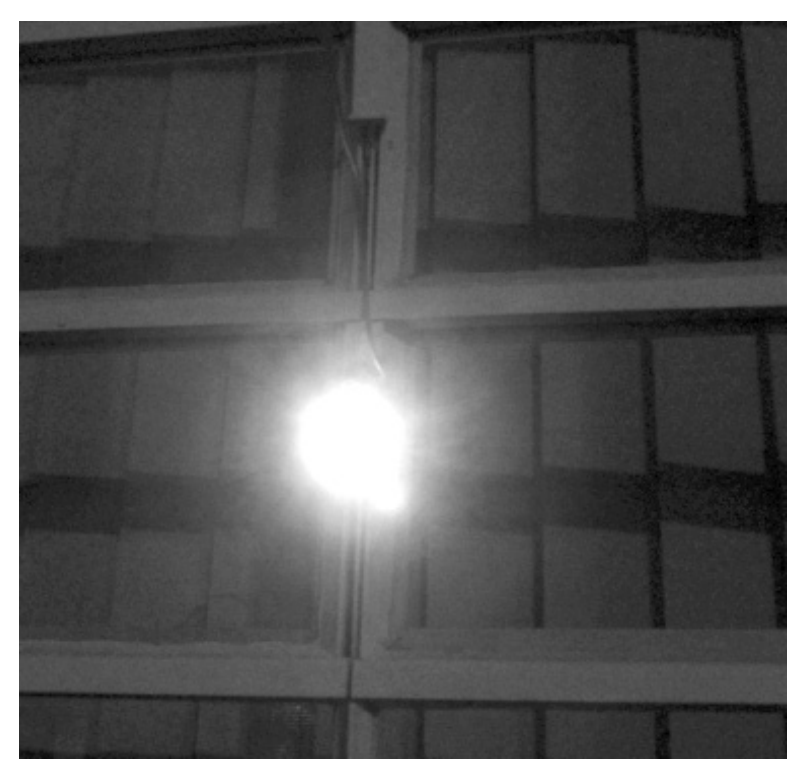

Figura 11 - exemplo de aplicação.

\section{CONCLUSÃO}

Pode-se concluir que o uso de motores de imã permanente obtidos de lixo eletrônico resulta em geração de energia elétrica diminuindo o impacto ambiental que esse tipo de resíduo provoca na natureza.

\section{REFERÊNCIAS}

REVISTA DO IDEC.Tecnologia que vira lixo, Revista do Idec, São Paulo, p. 26-30, 2009;

DIAS, V. S.; MARTINS, R. A.. Michael Faraday: $\mathrm{O}$ caminho da livraria à descoberta da indução eletromagnética. Ciência \& Educação, Bauru, v. 10, n. 3, p. 517-530, 2004;

SILVEIRA, F. L.; VARRIALE, M. C.. O rolamento freado do magneto na rampa: uma interessante aplicação da lei de Faraday-Lenz. Revista Brasileira de Ensino de Física, São Paulo, v. 31, n. 4, 4303 2009; 
SOUZA FILHO, M.P.; CHAIB, J.P.M.C.;

CALUZI; J.J. e ASSIS, A.K.T.. Revista Bra-

sileira de Ensino de Física, São Paulo, v. 29,

n. 4, p. 605-612, 2007;

WALKER, H. R. Fundamentos de Física 3 -

$4^{\circ}$ Ed. ,LTC Editora. 1998 